\title{
Sorción y solubilidad de materiales formulados con resina
}

\author{
Vaca $M \mathrm{~J}^{*}$, Ceballos $\mathrm{L}^{* *}$, Fuentes $\mathrm{MV}^{* * *}$, Osorio $\mathrm{R}^{* * * *}$, Toledano $\mathrm{M}^{* * * * *}$, \\ García-Godoy F*****
}

\section{RESUMEN}

El objetivo de este estudio fue determinar la sorción y solubilidad de cuatro resinas compuestas: Tetric Ceram (Vivadent), Ecusit (DMG), Degufill (Degussa) y Z-250 (3M-Espe); y dos resinas modificadas con poliácido: Luxat (DMG) e lonosit (DMG). Cinco especímenes circulares $(15 \mathrm{~mm} \times 0.85 \mathrm{~mm}$ ) de cada material fueron preparados y pulidos con discos de carburo de silicio. Los especímenes fueron desecados en una estufa a $37^{\circ} \mathrm{C}$ hasta que se registró un peso constante $\left(\mathrm{m}_{0}\right)$ y, posteriormente, se introdujeron en agua destilada a $37^{\circ} \mathrm{C}\left(\mathrm{m}_{1}\right)$. Una vez estabilizado su peso fueron desecados nuevamente, colocados en una campana al vacío $\left(60^{\circ} \mathrm{C}, 24 \mathrm{~h}\right)$ y pesados por última vez $\left(\mathrm{m}_{2}\right)$. El cálculo de la sorción (A) y solubilidad (S) se realizó mediante expresiones matemáticas interrelacionadas: $A=m_{1}-m_{2} / V ; S=m_{0}-m_{2} / V$. Los tests de ANOVA y Student Newman Keuls mostraron diferencias en la sorción y solubilidad según el material estudiado $(\mathrm{p}<0.05)$. Ionosit presentó los valores más altos de sorción y Tetric Ceram y Ecusit los más bajos. La sorción de Luxat fue más elevada que la de Degufill, pero la de ambos materiales fue similar a la del Z-250. La solubilidad de lonosit fue también significativamente más alta que la de los demás materiales. Las resinas compuestas evaluadas y Luxat presentaron unos valores de solubilidad similares.

Palabras clave: Sorción, solubilidad, resina compuesta, resina modificada con poliácido.

\begin{abstract}
The aim of this study was to determine the sorption and solubility of four resin composites: Tetric Ceram (Vivadent), Ecusit (DMG), Degufill (Degussa) and Z-250 (3M-Espe); and two polyacid-modified resins: Luxat (DMG) and lonosit (DMG). Five discs (15 mm x $0.85 \mathrm{~mm}$ ) of each material were prepared and ground wet to remove poorly polymerized suface layers. Discs were conditioned in a desiccator $\left(37^{\circ} \mathrm{C}\right)$ until a constant weight was achieved using an analytic balance $(\mathrm{m} 0)$; immersed in distilled water $37^{\circ} \mathrm{C}\left(\mathrm{m}_{1}\right)$ and finally into a vacuum oven $\left(60^{\circ} \mathrm{C}\right.$, $24 \mathrm{~h}$ ) and reweighted $\left(\mathrm{m}_{2}\right)$. Sorption $(A)$ and solubility $(S)$ were calculated: $A=m_{1}-m_{2} / V ; S=m_{0}-m_{2} / V$. ANOVA and SNK tests showed statistical differences among the studied materials. Ionosit showed the highest sorption and Tetric Ceram and Ecusit the lowest. Luxat presented higher sorption than Degufill, but both materials showed similar sorption values compared with Z-250. The higher solubility was obtained by lonosit. No statistical differences were found among resin composites and Luxat.
\end{abstract}

Key words: Water-sorption, solubility, resin composite, polyacid-modified resin.

* $\quad$ Estudiante de la Facultad de Odontología y Becaria de Investigación del Plan Propio de la Universidad de Granada.

** Becaria Post-doctoral del Plan Propio de la Universidad de Granada, adscrita al Dpto. de Estomatología.

*** Licenciada en Odontología.

**** Profesor Titular de Materiales Odontológicos. Facultad de Odontología. Universidad de Granada.

***** Profesor y Director de Investigación del College of Dental Medicine, Nova Southeastern University, Fort. Lauderdale, FL, Estados Unidos.

Vaca MJ, Ceballos L, Fuentes MV, Osorio R, Toledano M, García-Godoy F. Sorción y solubilidad de materiales formulados con resina. Av. Odontoestomatol 2003; 19-6: 283-289. 


\section{INTRODUCCIÓN}

Las mejoras en las propiedades de las resinas compuestas han permitido que sean el material restaurador actualmente más utilizado en dientes permanentes. Sin embargo, dadas las características específicas de la dentición primaria, otros materiales estéticos como son los cementos de ionómero de vidrio modificados con resina y las resinas compuestas modificadas con poliácidos, conocidas como compómeros, juegan también un papel importante (1).

Los cementos de ionómero de vidrio presentan algunas ventajas sobre las resinas compuestas como son su capacidad cariostática debida a su liberación de flúor y su adhesión química al tejido dentario $(2,3,4)$. No obstante, su manipulación, sus propiedades estéticas (4) y su resistencia mecánica (5) se han considerado inferiores a las de las resinas compuestas.

Los cementos de ionómero de vidrio fraguan como consecuencia de una reacción ácido-base entre un polvo de fluoroaluminosilicato básico y un ácido policarboxílico, reacción que necesita de la presencia de agua para producirse $(4,6)$.

Las resinas compuestas generalmente constan de una matriz orgánica polimerizable reforzada con partículas de relleno, fundamentalmente inorgánicas, y un agente de unión silano que conecta la porción orgánica y la inorgánica. La matriz resinosa contiene Bis-GMA, TEGDMA o UDMA, y el relleno está constituido normalmente por silicio amorfo, cuarzo, borosilicato, cristales de litio-aluminio-silicato y, para conseguir radiopacidad, se añaden cristales con óxidos de bario, estroncio o zinc $(1,4)$. El relleno se añade para controlar sus características de manipulación, disminuir la contracción de polimerización, aumentar su resistencia, y disminuir el desgaste abrasivo (4). La reacción de polimerización de la mayoría de las resinas compuestas disponibles en el mercado se produce por inducción lumínica.

Con el fin de aunar las propiedades ventajosas de los cementos de ionómero de vidrio y de las resinas compuestas se desarrollaron los cementos de ionómero de vidrio modificados con resina y las resinas modificadas con poliácidos $(5,7)$. En estos últimos materiales se combinan componentes del cristal de polial- quenoato, por ejemplo, cristales de fluorosilicato, con sustancias polimerizables de las resinas compuestas, como son los dimetacrilatos (8). De este modo, contienen los componentes esenciales de los cementos de ionómero de vidrio, pero falta su reacción de fraguado ácido-base típica por la ausencia de agua en su composición $(4,9)$. Por ello, presentan mejores propiedades mecánicas tempranas (10) y son más fáciles de manipular en clínica (8).

Todos estos materiales, una vez expuestos al medio oral, van a sufrir procesos de sorción y solubilidad en mayor o menor grado, que conllevan una degradación de los mismos, que afectará a la calidad y longevidad de las restauraciones (11).

La sorción de agua es un proceso de difusión controlada dentro de la matriz resinosa que puede conllevar su degradación y la ruptura de la unión entre el relleno y la matriz $(12,13)$. Además, se puede producir la liberación o disolución de partículas del relleno, iones y sustancias orgánicas, por ejemplo, monómeros residuales (14); este fenómeno produce una disminución del peso, y se denomina solubilidad (15).

Los procesos de sorción y solubilidad pueden provocar cambios dimensionales en los materiales (6), pueden afectar a sus propiedades mecánicas (14), pueden producir cambios en el color de las restauraciones, empeorando su aspecto estético (16), o alterar su biocompatibilidad (17), puesto que los componentes liberados podrían afectar a las células pulpares (18).

Dada la relevancia clínica de ambos procesos, el objetivo de este estudio fue cuantificar, in vitro, la sorción y solubilidad de distintos materiales formulados con resina disponibles en el mercado.

\section{MATERIAL Y MÉTODOS}

Para la realización de este estudio se prepararon 5 discos (15 mm de diámetro, $0.85 \mathrm{~mm}$ de espesor) de cada material utilizando unos moldes de teflón.

Los materiales que se evaluaron fueron cuatro resinas compuestas: Tetric Ceram (Vivadent, Liechtenstein), Ecusit (DMG, Hamburgo, Alemania), Degufill (Degussa Dental GmbH, Hanau, Alemania) y Z-250 
(3M-ESPE, St. Paul, MN, EE.UU.); y dos resinas modificadas con poliácido: Luxat (DMG, Hamburgo, Alemania) e Ionosit (DMG, Hamburgo, Alemania).

Los moldes se colocaron sobre una loseta de vidrio y el material de estudio se insertó procurando que no quedaran burbujas de aire atrapadas en su interior. Sobre éste se colocó otra loseta de vidrio con la que se hizo presión para conseguir una superficie lisa y un grosor homogéneo. Los discos fueron polimerizados utilizando una lámpara de luz halógena (Spectrum 800, Dentsply. Detrey Gmb H, Konstanz, Alemania). Cada diez exposiciones se comprobó que la intensidad de la luz sobrepasase los $600 \mathrm{mw} / \mathrm{cm}^{2}$ con un radiómetro de curado incorporado en la lámpara.

Transcurridos 20 minutos desde la polimerización, los discos fueron extraídos de los moldes y se pulieron con discos de carburo de silicio de 500, 800, 1000, 1200 y 4000 grits de forma consecutiva, con el fin de remover la capa de resina inhibida. Una vez pulidos se desecaron en una estufa a $37^{\circ} \mathrm{C}$ y se pesaron en una balanza analítica (AND HM-202 d=0.01/0.1 mg 210 $-1 \mathrm{mg}$. European Headquar-ters, Oxford, Reino Unido) colocada sobre una mesa antivibratoria. El peso de cada espécimen se determinó cada 24 horas hasta que alcanzó un valor constante. Este valor fue registrado y considerado como mo.

Tras esto, las muestras se sumergieron en agua destilada y se colocaron de nuevo en la estufa a $37^{\circ} \mathrm{C}$. Se registraron los pesos de los discos transcurridas 1 h, 6 h y 24 h sucesivamente y, a continuación se pesaron en intervalos de 48 horas hasta que su peso se estabilizó. Este valor se registró como m.

Las muestras se retiraron del agua y se colocaron de nuevo en la estufa a $37^{\circ} \mathrm{C}$ y se pesaron cada 48 horas hasta que su peso se hizo constante.

Por último se introdujeron en una campana de vacío a $60^{\circ} \mathrm{C}$, durante 24 horas para realizar la última pesada. Este valor fue registrado como $\mathrm{m}_{2}$.

Los valores de sorción y solubilidad se calcularon siguiendo las siguientes fórmulas:

$$
\begin{aligned}
& A=m_{1}-m_{2} / V \\
& S=m_{0}-m_{2} / V
\end{aligned}
$$

Donde:

A: sorción

S: solubilidad

mo es el peso registrado antes de colocar las muestras en agua

$\mathrm{m}_{1}$ es el peso registrado después del almacenamiento en agua

$\mathrm{m}_{2}$ es el peso registrado después del almacenamiento y desecación

Para la descripción de los datos se utilizó como medida de tendencia central la media aritmética y como medida de dispersión la desviación típica. Se analizaron mediante los tests de ANOVA y Student-NewmanKeuls aceptando un nivel de significación de $p<0.05$, con el paquete software SPSS/PC+7.5.2., W95/NT (SPSS Inc., Chicago, IL, Estados Unidos).

\section{RESULTADOS}

Las medias (x) y desviaciones típicas (dt) de los distintos materiales quedan reflejadas en la tabla 1.

El test de ANOVA puso de manifiesto la influencia del material evaluado en la variable dependiente, sorción $(F=4.494 ; p=0.003)$. Las comparaciones múltiples

\begin{tabular}{|c|c|c|c|c|c|c|c|}
\hline \multicolumn{7}{|c|}{ TABLA 1.- VALORES MEDIOS DE SORCIÓN Y SOLUBILIDAD OBTENIDOS CON LOS } \\
DIFERENTES MATERIALES EVALUADOS, EXPRESADOS EN $\mu \mathrm{gr} / \mathrm{mm}^{3}$ \\
\hline \\
\hline Sorción & $\mathrm{x}$ & $13.97 \mathrm{a}$ & $11.263 \mathrm{a}$ & $19.024 \mathrm{~b}$ & $22.232 \mathrm{bc}$ & $23.035 \mathrm{c}$ & $93.594 \mathrm{~d}$ \\
& $(\mathrm{dt})$ & $(0.817)$ & $(2.298)$ & $(3.668)$ & $0.791)$ & $(2.589)$ & $(3.114)$ \\
\hline Solubilidad & $\mathrm{x}$ & $1.526 \mathrm{a}$ & $2.329 \mathrm{a}$ & $2.731 \mathrm{a}$ & $0.339 \mathrm{a}$ & $3.935 \mathrm{ab}$ & $7.496 \mathrm{~b}$ \\
& $(\mathrm{dt})$ & $(0.994)$ & $(0.76)$ & $(1.94)$ & $(0.470)$ & $(1.367)$ & $(4.998)$ \\
\hline
\end{tabular}

Los resultados con la misma letra son estadísticamente similares $(p>0.05)$. 
con el test de Student-Newman-Keuls mostraron que lonosit presentaba valores de sorción estadísticamente superiores al resto. La sorción de Luxat fue significativamente más elevada que la de Tetric Ceram, Ecusit y Degufill, pero similar a la de Z-250. Degufill presentó una sorción estadísticamente más alta que la del Tetric Ceram y Ecusit, pero similar a la del Z250. Y, por último, Tetric Ceram y Ecusit presentaron un sorción estadísticamente inferior a la de los demás materiales evaluados.

Después de realizar el test de ANOVA $(\mathrm{F}=661.55$; $\mathrm{p}<0.0001)$ se comprobó también la influencia de la variable independiente, que en este caso se correspondía con el material estudiado, en la variable dependiente solubilidad. Una vez hechas las comparaciones múltiples con el test de Student-Newman-Keuls, se observó que la solubilidad determinada era estadísticamente superior en el caso de lonosit, comparada con la de Tetric Ceram, Ecusit, Degufill y Z-250, todos ellos resinas compuestas. Luxat sufrió un proceso de solubilidad en una magnitud intermedia, de tal forma que los resultados no eran diferentes a los presentados por el resto de los materiales estudiados.

\section{DISCUSIÓN}

Los resultados del presente estudio ponen de manifiesto la enorme influencia del tipo de material resinoso utilizado en los procesos de sorción y solubilidad que acontecen en condiciones de humedad. Los trabajos de investigación publicados evaluando dichos procesos son numerosos, sin embargo, la comparación de nuestros resultados con los de la mayoría de estos estudios no es tarea fácil debido a las diferencias en los materiales evaluados y la metodología utilizada (periodos de tiempo, modo de manipulación de los especimenes, tamaño de los mismos, medio de conservación y su $\mathrm{pH}$, técnicas de evaluación y análisis, etc) $(19,20,21)$. Por ello consideramos más interesante valorar los resultados obtenidos de un modo relativo que realizar una comparación estrictamente numérica.

El proceso de sorción fue mucho más importante y manifiesto en las resinas modificadas con poliácidos evaluadas que en las resinas compuestas, aunque en el caso del Luxat la diferencia no fue estadísticamen- te significativa respecto al Z-250. El fenómeno de sorción acontece fundamentalmente en la matriz resinosa, por tanto, la mayor fracción de matriz orgánica que presentan las resinas modificadas con poliácidos, en comparación con las resinas compuestas (22), podría justificar los resultados obtenidos. Además, y de acuerdo con Musanje et al. (20), hay otros factores que consideramos también importantes como son la hidratación de los núcleos de los cristales sensibles a la acción del ácido, así como la disolución necesariamente superior de la matriz resinosa de las resinas modificadas con poliácidos al ser más polares (23) e hidrofílicas.

La polimerización de las resinas modificadas con poliácidos lonosit y Luxat ocurre fundamentalmente por fotocurado, puesto que en su composición no contienen agua (4), imprescindible para que se produzca una reacción ácido-base. Sin embargo, una vez que entran en contacto con el medio oral la absorción de agua por parte de los mismos permitiría un fraguado más completo por reacción ácido-base. Esto es posible porque contienen metacrilatos modificados y monómeros bifuncionales con grupos ácidos carboxílicos que pueden reaccionar con los cationes liberados del cristal (23).

Esta reacción ácido-base se acompaña de una expansión higroscópica de fraguado, en la que reside una de sus principales cualidades, puesto que el leve aumento de su volumen compensaría la contracción de polimerización que el material sufre, mejorando el sellado marginal de las restauraciones (24). Sin embargo, la reacción ácido-base producida no supondría una mejora en las propiedades mecánicas del material, sino todo lo contrario, debido a la degradación de la unión entre la matriz y los cristales, a los efectos plastificantes de la sorción de agua y a la mayor labilidad de los enlaces creados, así como a la reducción en el volumen de la fracción cristalina original, que es más dura y rígida que los productos de la reacción ácido-base (20).

A pesar de que Luxat e Ionosit se clasifican como resinas modificadas con poliácidos, este último material presentó unos valores de sorción muy superiores. Hay varios factores que han podido influir en estas diferencias como son el menor contenido inorgánico de lonosit (14) y la presencia de TEDMA como dilu- 
yente del Bis-GMA en su composición, pues su incorporación como co-monómero diluyente implica una mayor sorción de agua (25).

También se observaron diferencias estadísticamente significativas en la sorción determinada para las diferentes resinas compuestas evaluadas, de tal modo que Tetric Ceram y Ecusit fueron menos sensibles a la sorción de agua que Degufill y Z250. A pesar de estas diferencias, los resultados entran dentro del rango de los publicados por otros autores $(11,26)$. Estas variaciones en la sorción pueden atribuirse a múltiples factores como son la composición química de la matriz resinosa (21). Por ejemplo, Z250 contiene en su composición TEGDMA que es el monómero que crea la red polimérica más densa y flexible, pero también la que más cantidad de agua absorbe (27). Además, aunque el relleno de por sí no absorbe agua, su composición química, proporción y el tamaño y forma de las partículas del mismo sí influyen en la sorción $(13,15,25,26)$, puesto que el agua absorbida se aloja en la interfase entre el relleno y la matriz polimérica, y una vez allí reacciona con el relleno o con el agente de unión $(1,28)$. Por lo tanto, la silanización del relleno también influye, puesto que cuanto más efectiva sea la unión entre las partículas de relleno y la matriz orgánica, menor será la sorción producida (28).

En relación a la solubilidad, las resinas modificadas con poliácidos, lonosit y Luxat, fueron más sensibles al proceso de solubilidad que las resinas compuestas evaluadas, aunque en el caso de Luxat las diferencias no fueron estadísticamente significativas.

Ionosit, al igual que ocurrió con la sorción, presentó una solubilidad significativamente más elevada que la otra resina modificada con poliácido y que las cuatro resinas compuestas evaluadas. Estos resultados ponen de manifiesto, la íntima relación entre los procesos de sorción y solubilidad, pues el agua absorbida, tras reaccionar con el agente de unión o con las partículas de relleno inorgánico, produce la separación de las partículas de relleno (o de sus componentes) contribuyendo a su liberación.

Las diferencias entre la solubilidad de Ionosit y Luxat pueden explicarse por sus diferentes composiciones químicas. Luxat contiene resinas basadas en UDMA que son menos solubles en agua que las que contienen Bis-GMA, monómero que incluye lonosit (29).
Este material también contiene TEDMA como diluyente, cuya solubilidad es mayor. Sin embargo, este monómero aporta fluidez al material lo que permite que se pueda aplicar más fácilmente en el fondo de las cavidades. Además, Inosit presenta un porcentaje mayor de cristales de Bario, más inestables que los de silicio (30). Estos cristales de álcalis y metales alcalino-térreos aumentan la degradación interna del material (13) pues al liberarse el Bario dentro de la restauración forman hidróxidos que al ir aumentando pueden incrementar localmente el $\mathrm{pH}$ dentro de la restauración. Un pH alto también afecta a la estabilidad del silano lo que conllevaría la mayor disolución de silicio y de los cristales de silicato (30).

Las diferencias entre ambos materiales justifican sus indicaciones clínicas distintas, a pesar de ambos están clasificados como resinas modificadas con poliácidos. La mayor sensibilidad del lonosit a la sorción y solubilidad supone también unas peores propiedades mecánicas (31), por lo que está indicado su uso exclusivamente como base cavitaria. Luxat al tener más relleno tiene mejores propiedades mecánicas y está diseñado para restaurar lesiones de tipo III y V en dientes deciduos. Sin embargo, ambos materiales comparten una propiedad que se considera beneficiosa respecto a las resinas compuestas y que está estrechamente relacionada con su mayor solubilidad, y es su capacidad para liberar iones flúor, por lo que se les atribuyen propiedades cariostáticas (8).

Las resinas compuestas evaluadas presentaron una solubilidad similar, concordando los valores con los descritos anteriormente $(11,26)$. Los resultados obtenidos fueron, en todo caso, inferiores a los de las resinas modificadas con poliácidos (7), pudiendo ser una de las causas su mayor grado de conversión y por tanto, a la menor presencia de monómeros sin reaccionar (17). Otros factores que influyen con el tamaño y composición de los componentes que se puedan desprender, la inhibición de la polimerización en las capas superficiales y el efecto de silanización del relleno (33). Puesto que la sensibilidad a la presencia de agua de las resinas compuestas es menor y poseen mejores propiedades mecánicas y estéticas, estos materiales están indicados para la restauración de cualquier tipo de lesión.

Podemos concluir que los resultados de este estudio 
demuestran que la sorción y solubilidad de agua son datos valiosos para entender el comportamiento clínico diferente de las resinas compuestas y las resinas modificadas con poliácidos. La sorción de agua afecta a las propiedades mecánicas de los materiales (26, 33), a su resistencia a la tensión (34), módulo de elasticidad (14) y resistencia al desgaste, así como a las propiedades ópticas de estos materiales (16). Por otro lado, la liberación de monómeros sin reaccionar puede estimular el crecimiento bacteriano alrededor de las restauraciones y favorecer la aparición de reacciones alérgicas en algunos pacientes (17). Por tanto, todos estos factores deberían tenerse en cuenta, además de su adhesión al esmalte y la dentina, y la liberación de flúor, cuando se elige un material en clínica.

Por último, cabe destacar que los resultados obtenidos en este trabajo in vitro no pueden extrapolarse directamente a la situación clínica, puesto que está demostrada la mayor solubilidad de los materiales en saliva que en agua destilada (35), sin embargo, aportan una información valiosa sobre el comportamiento relativo de unos materiales respecto a otros.

\section{AGRADECIMIENTOS}

Al plan propio de la Universidad de Granada que concedió las becas de Iniciación a la Investigación y la beca postdoctoral.

Los autores agradecen a Gertrudis GómezVillaescusa su ayuda en la preparación de los especimenes. Este trabajo fue financiado por el Proyecto de Investigación CICYT/FEDER MAT 2001-2843-CO2 Y RED CYTED VIll. J.

\section{BIBLIOGRAFÍA}

1. Geurtsen W. Substances released from dental resin composites and glass ionomer cements. Eur J Oral Sci 1998; 106: 687-95.

2. Swift EJ, Pawlus MA, Vargas MA. Shear bond strengths of resin-modified glass-ionomer restorative materials. Oper Dent 1995; 20: 138-43.

3. Woolford MJ, Grieve AR. Release of fluorid from glass polyalkenoate (ionomer) cement subjected to radiant heat. J Dent 1995; 23: 233-7.

4. Kugel G. Direct and indirect adhesive restorative materials: A review. Am J Dent 2000; 13: 35D-40D.

5. Sidhu S.K, Watson T.F. Resin-modified glass ionomer materials. Am J Dent 1995; 8: No.1. 59-67.

6. Naasan M.A, Watson.T.F. Conventional glass ionomers as posterior restorations. Am J Dent 1998; 11: No $1.36-45$.

7. Iwami Y, Yamamoto H, Sato W, Kawai K, Torii $M$, Ebisu S. Weight change of various light-cured restorative materials after water immersion. Oper Dent 1998; 23: 132-7.

8. Geurtsen W, Leyhausen G, García-Godoy F. Effect of storage media on the fluoride relase and surface microhardness of four polyacid-modified composite resins ("compomers"). Dent Mater 1999; 15: 196201.

9. McLean JW, Nicholson JW, Wilson AD. Proposed nomenclature for glass-ionomer dental cements and related materials. Quintessence Int 1994; 25: 587-9.

10. Attin T, Vataschki M, Hellwig E. Properties of resinmodified glass-ionomer restorative materials and two polyacid-modified resin composite materials. Quintessence Int 1996:27: 203-9.

11. Ortengren U, Andersson F, Elgh U, Terselius B, Karlsson S. Influence of $\mathrm{pH}$ and storage time on the sorption and solubility behaviour of three composite resin materials. J Dent 2001; 29: 35-41.

12. Braden M, Clarke RL. Water absorption characteristics of dental microfine composite filling materials. Biomater 1984; 5: 369-72.

13. Söderholm KJ, Zigan M, Ragan $M$ et al. Hydrolytic degradation of dental composites. J Dent Res 1984; 63: $1248-54$.

14. Oysaed H, Ruyter IE. Composites for use in posterior teeth: mechanical properties tested under dry and wet conditions. J Biomed Mater Res 1986; 20: 261-71. 
15. Fan PL, Edhal A, Leung RL, Stanford JW. Alternative interpretations of water sorption values of composite resins. J Dent Res 1985; 64: 78-80.

16. Söderholm KJ. Leaking of fillers in dental composites. J Dent Res 1983; 62: 126-30.

17. Ferracane JL. Elution of leachable components from composites. J Oral Rehabil 1994; 21: 441-52.

18. Ferracane JL, Condon JR. Rate of elution of leachable components from composite. Dent Mater 1990; 6: 282-7.

19. Small ICB, Watson TF, Chadwick AV, Sidhu SK. Water sorption in resin-modified glass-ionomer cements: An in vitro comparison with other materials. Biomater 1998; 19: 545-50.

20. Musanje L, Shu M, Darvell BW. Water sorption and mechanical behaviour of cosmetic direct restorative materials in artificial saliva. Dent Mater 2001; 17: 394-401.

21. Pearson GJ. Long term water sorption and solubility of composite filling materials. J Dent 1979; 7: 64-8.

22. Milleding P, Ahlgren F, Wennerberg A, Örtengren U, Karlsson S. Microhardness and surface topography of a composite resin cement after water storage. Int J Prosthodont 1998; 1: 21-6.

23. Meyer JM, Cattani-Lorente MA, Dupuis V. Compomers: between glass ionomer cements and composites. Biomater 1998; 19: 529-39.

24. Torstenson B, Brännström M. Contraction gap under composite resin restorations. Effect of hygroscopic expansion and thermal stress. Oper Dent 1988; 13: 24.

25. Braden M, Davy KWM. Water absortion characteristics of some unfilled resins. Biomater 1986; 7: 474-5.

26. Oysaed H, Ruyter IE. Water sorption and filler characteristics of composites for use in posterior teeth. J Dent Res 1986; 65: 1315-8.

\section{Sideridou I, Tserki V, Papanastasiou G. Study of}

water sorption, solubility and modulus of elasticity of light-cured dimethacrylate-based dental resins. Biomater 2003; 24: 655-65.

28. Kalachandra S, Wilson TW. Water sorption and mechanical properties of light-cured proprietary composite tooth restorative materials. Biomater 1992; 13: 105-9.

29. Pearson GJ, Longman CM. Water sorption and solubility of resin-based materials following inadequate polymerisation by a visible-light curing system. J Oral Rehabil 1989; 16: 57-61.

30. Sarkar NK. Internal corrosion in dental composite wear: Its significance and simulation. J Biomed Mater Res 2000; 53: 371-80.

31. Ferracane JL, Hopkin JK, Condon JR. Properties of heat-treated composites after aging in water. Dent Mater 1995; 11: 354-8.

32. Mohsen NM, Craig RG. Hydrolytic stability of silanated zirconia-silica-urethane dimethacrylate composites. J Oral Rehabil 1995; 22: 213-20.

33. El-Hadary A, Drummond JL. Comparative study of water sorption, solubility, and tensile bond strength of two soft lining materials. J Prosthet Dent 2000; 83: 356-61.

34. Söderholm KJM, Roberts MJ. Influence of water exposure on the tensile strength of composites. J Dent Res 1990; 69: 1812.

35. Söderholm KJM, Mukherjee R, Longmate J. Filler leachability of composites stored in distilled water or artificial saliva. J Dent Res 1996; 75: 1692-9.

\section{CORRESPONDENCIA}

Dra. Laura Ceballos

C/ Emperatriz Eugenia 19, 6 D

18003 Granada

e-mail: lauraceballos@hotmail.com

TIf: 958372524 